\title{
Unidad Didáctica Adaptada para la Educación Física en un Centro de Educación Especial
}

\section{Adapted Didactic Unit for Physical Education in a Special Education Centre}

\author{
Khaled Malas Tolsá1, Olga Malas Tolsáa y Meritxell Ribas Grebol ${ }^{3}$ \\ 1 C.E.E. Font de l'Abella-Girona \\ 2 Departament de Psicología y Pedagogía - Facultad de Ciencias de la Educación, Psicología y Trabajo Social. UdL - Lleida \\ 3 C.E.P. Les Debeses - Salt (Girona)
}

\begin{abstract}
Resumen: En este trabajo se procede a describir una Unidad Didáctica Adaptada, sobre el bloque de contenidos "El Cuerpo: Imagen y Percepción“, perteneciente a un programa de Educación Física para los alumnos de un centro de educación especial con niños afectados, en diferentes grados, de Discapacidad Intelectual, Trastorno Generalizado del desarrollo y Plurideficiencia. El propósito de este artículo es dar a conocer una experiencia exitosa a otros centros de educación especial de características similares. Palabras clave: Educación física adaptada; Educación especial; El cuerpo: imagen y percepción; Discapacidad intelectual; Trastorno generalizado del desarrollo; Plurideficiencia.
\end{abstract}

Abstract: In this work we proceed to describe an Adapted Didactic Unit, on the content block "The Body: Image and Perception", belonging to a Physical Education program for the students of a special education centre with affected children, in different degrees, of Intellectual Disability , Generalized Developmental Disorder and Multidisability. The purpose of this article is to present a successful experience to other special education centres with similar characteristics.

Key words: Adapted physical education; Special education; The body: image and perception; Intellectual disability; Generalized development issue; Multidisability.

\section{Introducción}

La actividad ha sido diseñada para un Centro de Educación Especial con alumnos afectados por Discapacidad Intelectual, Trastorno Generalizado del desarrollo y Plurideficiencia, que debido a sus particularidades individuales no pueden ser atendidos en la escuela ordinaria.

Estos alumnos, a pesar de ser atendidos en los mismos centros y formar parte de grupos clase comunes, presentan particularidades propias y muy diferenciadas. Así, la Discapacidad Intelectual se caracteriza por limitaciones significativas tanto a nivel intelectual, con sus diferentes grados en función del coeficiente intelectual, como a nivel de adquisición de conductas adaptativas y habilidades conceptuales, sociales y prácticas (Luckasson et al., 2002; Alonso, 2003). Por su parte, los Trastornos Generalizados del Desarrollo, se refieren a un amplio grupo de trastornos cognitivo-conductuales, con grados variables de severidad, tales como el trastorno autista, el síndrome de Asperger, el síndrome de Rett, el trastorno desintegrativo infantil y el trastorno generalizado del desarrollo no especificado o autismo atípico. Estos trastornos se hallan ligados a múltiples causas de naturaleza biológica, que aparecen durante el desarrollo infantil, y que entre otros síntomas, dan lugar a problemas de socialización y de la comunicación verbal y no verbal, que originan patrones de conducta restrictivos y repetitivos (Iudicibus. 2011). Por último, las Plurideficiencia se caracterizan por la combinación de una deficiencia

Dirección para correspondencia [Correspodence address]: Olga Malas Tolsá. E-mail: olga.malas@hotmail.com mental con un déficit sensorial y/o motor que limita la percepción y la capacidad comunicativa con el entorno, restando autonomía y capacidad para satisfacer las necesidades básicas (Segura, 2011). Ello hace que, aunque presenten algún síntoma común, cada caso sea diferente.

Revisando las notas y consejos emitidos por las diferentes instituciones educativas, sabemos que como profesionales encargados de atender y formar a estos niños debemos aplicar, durante nuestras clases, programas curriculares diferenciados y/o adaptados a cada caso, priorizando los contenidos que hacen referencia a la adquisición de la autonomía personal y social, el desarrollo de las actitudes y capacidades de comunicación interpersonal y la adquisición de hábitos, destrezas y conocimientos básicos que les ayuden a la comprensión de la realidad más inmediata, dentro de las posibilidades de cada uno de los alumnos.

A la hora de hacer la adaptación curricular nos hemos encontrado con que hay bastantes publicaciones que hablan sobre estos trastornos a nivel clínico, psicológico y de intervención (Novell et al., 2003; Ancona et al., 2016; Barron y Castilla, 2016; Llario et al., 2016), pero son muy escasas las publicaciones sobre adaptación de unidades didácticas para la Educación Física destinadas a estos colectivos. Ello hace que cualquier propuesta deba ser analizada de forma previa, durante y tras su aplicación, para adaptarla y para proponer opciones paralelas que faciliten la inclusión de los alumnos que conforman el grupo clase.

A la hora de diseñar una propuesta debemos tener en cuen- 
ta que, tanto los niños afectados de Trastorno Generalizado del Desarrollo, como de Discapacidad Intelectual o Plurideficiencia, precisan de un refuerzo positivo constante, por lo que las actividades planificadas para cada sesión deben ser tales que permitan mantener su atención y ser asumibles por todos ellos, con objetivos y resultados inmediatos, concretos, observables y valiosos para la persona que los logra (Utley, 1994; Kelly, 2006; Sanz y Reina, 2012). Para ello tenemos que prever alternativas que nos permitan adaptarnos a cada caso y situación. Es decir, necesitamos diseñar muchas actividades y tantas variables de las mismas como casos y situaciones nos podemos encontrar pues no podemos dejar que ninguno de ellos sienta que ha fracasado en los objetivos planteados. Lamentablemente, según la escasa información hallada, este colectivo tiene unas características que limitan en gran medida la experimentación. Estos nińos, en general, acostumbran a tener problemas de comprensión y se desconciertan con mucha facilidad. Por su parte, los niños afectados de trastorno generalizado del desarrollo no responden bien a condiciones de aprendizaje basadas en técnicas de ensayo y error, pues los errores los sumen en el desconcierto y el negativismo; y es necesario prever y anticipar los cambios para evitar que los vivan como experiencias desconcertantes o terroríficas (Fernández, 2011; Iudicibus, 2011). Todo esto hace imprescindible el poder contar con casos de experiencias previas exitosas en este campo.

Por ello, el objetivo de este artículo es dar a conocer una Unidad Didáctica Adaptada exitosa tras la aplicación en un grupo heterogéneo de niños afectados de discapacidad intelectual.

\section{Marco teorico}

Se elige adaptar una Unidad Didáctica para el bloque de contenidos "El cuerpo: imagen y percepción" porque en base a la información clínica es habitual que estos niños presenten retrasos o problemas psicomotrices relacionados con la coordinación de movimientos, posturales o espacio-temporales, todos ellos relacionados con la construcción del esquema corporal (García y Ruiz, 2001; Gento, 2011).

El esquema corporal es el conocimiento inmediato y continuo que tenemos de nuestro cuerpo, tanto en estado estático como en movimiento, en relación con sus diferentes partes, con el espacio y con los objetos que nos rodean; de tal modo que un esquema corporal mal estructurado dará lugar a una descoordinación de movimientos o una incorrecta actitud postural, a desajustes espacio-temporales, a inseguridad y agresividad en las relaciones con los otros y a dificultades en otras áreas de conocimientos que predispondrán al fracaso escolar (Le Boulch, 1987).

Por su parte, la asignatura de Educación Física, con respecto a otras, incorpora actividades de motricidad y de per- cepción espacio-temporal que pueden ser de gran ayuda para trabajar este bloque de contenidos curriculares, pudiendo incorporar además objetivos didácticos que permitan el desarrollo de competencias relacionadas tales como el lenguaje y la socialización.

La Unidad Didáctica se centra el las partes del cuerpo. Se selecciona teniendo en cuenta la información aportada por Le Boulch (1987), según la cual la base de partida para una adecuada construcción del Esquema Corporal es el conocimiento y control del propio cuerpo, para en una segunda fase poder utilizarlo en todas sus dimensiones. Por eso, según este autor, lo más importante en el desarrollo del esquema corporal es conocer y sentir las diferentes partes del mismo; para posteriormente, desarrollar diferentes acciones encaminadas a trabajar la disociación de movimientos (independencia de brazos y piernas con respecto al tronco), a reconocer y representar el cuerpo de los demás, al control sobre la postura y la respiración y a la capacidad de relajar todas o partes de su cuerpo.

A la hora de adaptar la actividad y proponer variantes que cubran al mayor número posible de alumnos, se han tenido también en cuenta los consejos de Castañer y Camerino (1991) y Arteaga et al. (1997), que recomiendan:

- En primer lugar, aprovechar el juego para el aprendizaje del nombre y funcionalidad de las distintas partes de su cuerpo y el conocimiento de sus posibilidades corporales.

- Seguidamente trabajar con el equilibrio estático (mantener una postura) para ir incorporando el equilibrio dinámico (mantener una posición en desplazamiento) y de re-equilibración (mantener una posición equilibrada estática después de una dinámica, como cuando se realiza un salto).

- Habiendo alcanzado los puntos primero y segundo es posible empezar a trabajar con la actitud postural, la tonicidad y la lateralidad (reconocer los dos lados corporales, adquirir una adecuada orientación y percepción espacial y adquirir una adecuada coordinación del cuerpo).

- Para finalmente trabajar la respiración y la relajación lo cual demanda una mayor atención hacia aquello que ocurre dentro de su cuerpo, proporcionando pautas tendentes a lograr la distensión psíquica, la eliminación de ansiedades, timideces, bloqueos, etc., que facilitará un mayor bienestar corporal.

Con respecto el desarrollo de la percepción y estructuración espacial y temporal seguiremos las recomendaciones de Castañer y Camerino (1991). Según este autor, el tratamiento del espacio permite visionar su ocupación y la orientación en función de la lateralidad y el ritmo inherente a cada uno de los movimientos que se realizan, tanto voluntarios como 
involuntarios y que permite una mayor coordinación y un aprendizaje más eficaz. Por su parte el desarrollo de la percepción y estructuración temporal se desarrolla paralelamente a las adquisiciones espaciales, ya que a partir de las informaciones espaciales el niño adquiere las nociones de duración e intervalo, apreciación de trayectorias, velocidades, anticipación, reproducción de estructuras y secuencias rítmicas, etc.

\section{Propuesta didáctica}

\section{Título de la unidad didáctica}

Las Partes Del Cuerpo

\section{Contextualización}

Esta Unidad Didáctica va dirigida a un grupo de alumnos de una Escuela de Educación Especial. El grupo esta formado por niños y nińas de 10 a 13 años de edad con necesidades educativas especiales derivadas de discapacidad intelectual (DI) en diferentes grados, trastornos del aspecto autista (TEA) en diferentes grados y Plurideficiencias (discapacidad psíquica, sensorial y/o motriz).

La duración de la unidad didáctica es de 3-4 sesiones, con una duración de entre 50 y 60 minutos cada una de ella, a aplicar semanalmente, en la mitad del primer trimestre del curso, a lo largo de 3 semanas, que se concretan en 3 sesiones llevadas a cabo una vez por semana.

La experiencia se ha llevado a cabo con tres grupos de 1012 alumnos. Para que la sesión tenga éxito es importante poder tener algún soporte, ya sea un educados, un maestro, u otros profesionales. De esta manera los alumnos reciben más ayudas y refuerzos. Los tres grupos son bastante heterogéneos en cuanto al nivel de competencias, intereses y aprendizajes.

Cabe destacar que dentro del grupo de ensayo hemos contado con algunos alumnos que no utilizan el lenguaje como medio de comunicación, sino que se expresan con sonidos, imágenes o signos. Otros presentan un nivel muy bajo de comprensión y se guían por la imitación.

\section{Justificación de la elección}

Se elige adaptar esta Unidad Didáctica al observar que estos alumnos presentaban, a distintos niveles, problemas en cuanto a la coordinación de movimientos, posturales o espacio-temporales, relacionados con la construcción del esquema corporal.

El esquema corporal es el conocimiento inmediato y continuo que tenemos de nuestro cuerpo. Por ello en esta adaptación se presentan diferentes actividades donde los alumnos trabajan a partir de las partes del cuerpo, utilizando diferentes materiales y técnicas. En todas las actividades, se presentan variantes que vienen determinadas por el nivel de comprensión de los alumnos y por sus capacidades físicas e intelectuales.

La Unidad y los aspectos a trabajar se presentan como un continuo y el alcance lo determina la capacidad de cada alumno. Se parte del conocimiento de las partes del cuerpo y su funcionalidad. A partir de hay, de todos los elementos relacionados con la Educación Física y la Expresión Corporal buscaremos trabajar aspectos relacionados con la coordinación general, el equilibrio, la tonicidad, el ritmo, la respiración y la coordinación visomotriz. Con aquellos niños/as que ya conocen las diferentes partes del cuerpo y su vocabulario se llega a trabajar la lateralidad y los conceptos derecha/izquierda.

\section{Objetivos}

Con nuestro trabajo pretendemos mejorar las habilidades motrices de estos niños/as, así como sus habilidades comunicativas y sociales, su adaptación al entorno, la reducción de conductas inadecuadas y la adquisición de seguridad en sí mismos a fin de que sean lo más autónomos posibles dentro de las posibilidades de cada uno de los alumnos.

A fin de conseguirlo, se plantean los siguientes Objetivos didácticos:

1. Vivenciar las partes del cuerpo (Identificar y tomar conciencia de los diferentes segmentos corporales: Cabeza, tronco, brazos y piernas).

2. Trabajar el vocabulario de las partes del cuerpo.

3. Demostrar interés por la sesión.

4. Mostrar hábitos de higiene durante y al finalizar la sesión.

5. Trabajar la socialización mediante el trabajo cooperativo con los compañeros o los adultos presentes durante las sesiones.

\section{Contenidos de la unidad didactica}

La Unidad Didáctica incluye los siguientes contenidos:

\section{Conceptuales:}

- Conocimiento del propio cuerpo

- Trabajo del vocabulario de las partes del cuerpo

- Percepción del espacio y del tiempo.

Procedimentales:

- Observación y experimentación de movimientos.

- Habilidades manipulativas y coordinaciones visomanuales.

\section{Actitudinales:}

- Respeto y cuidado del propio cuerpo.

- Autonomía y confianza en sus posibilidades de movimiento.

- Actitud de ayuda y cooperación con los compañeros y adultos.

- Normas básicas de higiene y cuidado corporal. 


\section{Competencias relacionadas}

Con esta Unidad Didáctica se trabajan:

- Competencia lingüística.

- Competencia social

- Aprender a aprender

- Conciencia y expresiones culturales.

\section{Atención a la diversidad.}

Durante la realización de las diferentes sesiones se han tenido presente las estrategias de aprendizaje y las adaptaciones curriculares y metodológicas necesarias para poder ofrecer todas las ayudas necesarias a los alumnos.

El objetivo debe ser proporcionar a cada alumno la respuesta que necesita en función de las necesidades y también de sus límites, tratando siempre que la respuesta los aleje lo menos posible de las que son comunes para el resto.

Basándonos en las recomendaciones y experiencias de los autores mencionados a lo largo de este artículo y en la experiencia personal previa, sobre estrategias de aprendizaje y adaptaciones curriculares y metodológicas, durante la realización de las diferentes sesiones se ha procedido a adaptar las actividades mediante la aplicación de las siguientes estrategias:

1. Atención individualizada, tanto en la información inicial como el conocimiento de la ejecución y los resultados.

A fin de incluir niños con discapacidad auditiva o con problemas de atención (muy habituales en estos grupos), a la hora de hacer las explicaciones se comprueba que todos ellos esta de cara y pueden ver fácilmente a quien hace la explicación. En segundo lugar, a fin de asegurar la comprensión, los adultos escenifican un ejemplo y durante su ejecución se va explicando la actividad (comunicación auditiva y visual).

\section{Pocas reglas y normas en las actividades.}

Muchos de estos niños tienen una capacidad de comprensión limitada. Por ello, la sesión se fragmenta en actividades pequeñas. Las actividades se planifican de modo que resulten sencillas y no estén sujetas a más de una o dos normas. Las explicaciones se hacen actividad por actividad.

3. Utilizar un vocabulario adecuado al nivel de comprensión.

En todo momento se usa un lenguaje claro y sencillo. Expresiones concretas y breves. Evitando explicaciones largas que los aburren y/o no comprenden.
4. Siempre se buscará la seguridad, evitando la lesión.

La planificación se efectúa teniendo en cuenta los riesgos a partir de la experiencia previa con grupos similares y durante la ejecución se ha previsto la colaboración del maestro con un educador de apoyo, un fisioterapeuta o otro profesional.

5. Facilitación de tareas mediante ayudas verbales, visuales y táctiles.

En todas las actividades se tienen presente los problemas de comprensión. Es por eso que tras la explicación general y la dramatización se procede a explicar la actividad individualmente a quien lo necesita. En caso necesario se prevé el uso de pictogramas y hojas con ejemplos, para lo cual se tiene a mano hojas grandes y rotuladores de colores.

6. Variantes en todas las actividades para dar la opción de participar a todos/as.

La finalidad es que no haya ni ganadores ni perdedores. Como base de partida la actividad tiene un objetivo mínimo alcanzable por todos. En caso contrario se descarta y se hace otra cosa. Para aquellos alumnos con mayores dificultades de logro se prevé la ayuda de un adulto a fin de que lo consiga.

La sesión se irá adaptando, en cuanto a logros, a cada niño/a teniendo en cuenta su estado fisiológico y psicológico en el momento de la clase, sin perder de vista el objetivo principal (mejora de la expresión corporal y de actitud social) sobre los objetivos de la actividad que en ese momento se esta llevando a cabo.

\section{Mayor utilización de reforzador positivos.}

En todo momento se anima al grupo con expresiones positivas. Los mensajes son de apoyo para lograr el objetivo y de felicitación una vez finalizada la experiencia, independientemente de hasta donde han sido capaces de llegar. No hay lugar para refuerzo negativo.

8. La colaboración entre los compañeros de su propio grupo.

Se pide a los alumnos que ayuden a los demás (actividad por parejas de alumnos o alumno/tutor). Con ello se pretende incrementar las actitudes colaborativas y socializadoras (empatía entre compańeros, impulso de ayudar y pedir ayuda, ...)

\section{Prioridad en algunos objetivos y contenidos.}

Se prioriza la acción socializadora, seguida de aquella que facilita la expresión corporal sobre otros contenidos curriculares. 
10. Modificación en el tiempo de consecución de las actividades.

No se han establecido tiempos de finalización. La actividad socializadora es constante y progresiva en tanto que los logros en expresión corporal y otros objetivos transversales presentan tiempos y alcances variables que no son forzados en ningún momento.

\section{Metodologia}

Tras analizar las metodologías recomendadas o utilizadas en sus estudios por Antequera et al. (2008), Amate y Ordás (2012), Sos et al. (2013), Alba y Díaz (2016), Márquez y Celis (2016) y Pérez y Martín (2016) se decide seguir una metodología basada en el mando directo y la asignación de tareas, el descubrimiento guiado, el trabajo cooperativo y la experimentación libre.

En cada momento se selecciona la metodología a usar ha dependido de los objetivos competenciales, del grado de deficiencia de los alumnos, de su estado emocional y de los espacios y materiales disponibles. Es decir, siguiendo las recomendaciones de Alós (1997), se tiene en cuenta la gran heterogeneidad de los grupos por lo que se recurre a un conjunto de metodologías, aplicando las mas adecuada a cada caso y momento a fin de dar respuesta a la singularidad e individualidad y a la naturaleza dinámica y cambiante de cada alumno a lo largo de la sesión. Es por ello que se recurre de forma continuada a una variada gama de enfoques metodológicos, seleccionando una vía de actuación u otra a partir del conocimiento previo de cada alumno, donde puede llegar y que podemos esperar de él con respecto a la actividad y sus objetivos, y cuales pueden ser sus respuestas en diferentes contextos a fin de favorecer su socialización mediante la expresión de respuestas positivas y el control de las respuestas negativas.

En general, las sesiones se inician siguiendo metodologías de mando directo y asignación de tareas, con instrucciones basadas en la ejemplificación y posterior imitación y repetición. Las consignas dadas a los alumnos son breves, claras y sencillas. El trabajo cooperativo y el descubrimiento guiado se trabajan principalmente de forma paralela a metodologías de mando directo. Las sesiones se finalizan con metodologías basadas en la experimentación libre.

Teniendo en cuenta las limitaciones en la capacidad comunicativa de algunos de los alumnos, se utilizan canales de comunicación auditivos, visuales y táctiles, de modo que los niños reciban mensajes informativos, afectivos y empáticos. También se intenta establecer una comunicación metacomunicativa, más que educativa, ya que en este caso lo que más nos interesa es enseńar a comunicar, mejorar el lenguaje tanto verbal como corporal (expresión corporal).

Para llevar a cabo las actividades utilizamos globos y aros.
El uso de estas herramientas en actividades de este tipo en habitual en escuelas ordinarias (Garcia y Ruiz, 2001; Castro, 2008). Se eligen en base a las capacidades psicológicas y motrices del alumnado con el que vamos a intervenir.

Con la intención de que los alumnos se sientan seguros y asocien la asignatura y su funcionamiento, en las sesiones siempre se sigue la siguiente estructura:

- Parte inicial: Se da la bienvenida a los alumnos, se mira quien falta y se les pregunta cómo están y/o se habla de algo que alguno de ellos nos quieran contar. A continuación se pasa lista y se comprueba que todos llevan la ropa de deporte y la bolsa de higiene personal.

- Desarrollo de la actividad: Se hace una explicación breve y concisa de lo que se va a hacer y por qué. A continuación se hace una ejemplificación para pasar al desarrollo y adquisición de los contenidos.

- Parte final: Vuelta a la calma. Comentamos la sesión y después nos trasladamos a los vestuarios donde se sigue con la adquisición de los hábitos de higiene (ducha y cambio de ropa).

\section{Desarrollo de las sesiones}

Sesión: 1

Duración: 50/60 minutos.

Equipo docente: Un maestro y un monitor.

Alumnos: Grupo de 10-12 alumnos.

Espacio: Pista, sala o pabellón.

Material: Globos. Dispondremos de globos sin hinchar. Pero además, antes de inicial la clase hincharemos tantos globos como alumnos.

Organización: Individual.

\section{Desarrollo:}

1. Los participante, de pie o sentados, formarán un semicírculo, todos de cara al maestro.

a. Variante: Si alguno de los niños no tiene capacidad para alinearse en el semicírculo, le ayudará un monitor. Si alguno no puede estar de pie se colocará sentado.

2. Se presenta a los alumnos un globo y se les pregunta si saben lo que es, si alguna vez han jugado con ellos y si han hinchado alguno.

3. Les dejamos uno de muestra para que lo miren, lo toquen y experimenten con él.

a. Variante: Si alguno de los nińos no tiene capacidad para hacerlo solo le ayudara un monitor (poniéndoselo en la mano, pasándole la mano, etc.)

4. Si algún alumno del grupo sabe inflar el globo le decimos que pruebe a hincharlo.

a. Variante: Antes de darles los globos para que los hinchen se analizan las capacidades individuales y 
los riesgos que puede tener. Si en el grupo hay riesgo de que algún alumno pueda ingerir el globo se omite este punto y se les presentan los globos ya hinchados. Lo primordial es crear un ambiente seguro.

5. Cuando cada niño y niña tiene su globo hinchado, les dejamos que lo miren, lo toquen y experimenten con él. a. Variante: Si alguno de los niños no tiene capacidad para hacerlo solo le ayudara un monitor (poniéndoselo en la mano, pasándole la mano, etc.)

6. Si el grupo o gran parte del grupo tiene un buen nivel de comprensión, se les pide que se dispersen por la sala o pista con su globo, para que tengan más libertad de movimiento.

a. Variante: Si alguno de los niños no tiene capacidad para realizar esta tarea desplazándose por la pista, la mejor solución es que todos se sienten en el suelo en forma de semicírculo.

7. Se les pide que intenten colocarse el globo en diferentes partes del cuerpo sin que se les caiga al suelo. El maestro ira diciendo las diferentes partes del cuerpo (por ejemplo, en la cabeza, en la mano, en el pie, en la espalda, etc) y al mismo tiempo hará una escenificación para que puedan imitarlo.

a. Variante 1: Si alguno de los niños no tiene capacidad para realizar esta tarea, se les pedirá que solo se toquen con el globo en la cabeza, la mano, la pierna, la espalda, etc.

b. Variante 2: Si alguno de los niños no tiene capacidad para realizar la tarea según la Variante 1, entonces uno de los tutores procederá a tocarle con el globo en la cabeza, la mano, la pierna, la espalda, etc. complementando la actividad con mas tareas de experimentación con el globo en cooperación con sus compańeros y adulto en forma de compartirlos y pasarlos entre ellos.

c. Variante 3: Con aquellos niños/as que ya conocen las diferentes partes del cuerpo y su vocabulario se intenta trabajar la lateralidad y los conceptos derecha/izquierda (brazo derecho/izquierdo, pierna derecha/izquierda).

8. Para acabar la sesión pedimos que coloquen los globos en una caja y que formen nuevamente el semicírculo, para poder felicitarlos por lo bien que lo han hecho.

9. Procedemos con los hábitos de higiene generales establecidos para después de cada clase.
Sesión: 2

Duración: 50/60 minutos.

Equipo docente: Un maestro y un monitor.

Alumnos: Grupo de 10-12 alumnos.

Espacio: Pista, sala o pabellón.

Material: Aros de plástico.

Organización: Individual.

\section{Desarrollo:}

1. Los participante, de pie o sentados, formarán un semicírculo, todos de cara al maestro.

a. Variante: Si alguno de los niños no tiene capacidad para alinearse en el semicírculo, le ayudará un monitor. Si alguno no puede estar de pie se colocará sentado.

2. Se presenta a los alumnos un aro de plástico y se les pregunta si saben lo que es y si alguna vez han jugado con un aro.

3. Se le da un aro a cada uno y se les deja unos minutos para que lo miren, lo toquen y experimenten con él.

a. Variante: Si alguno de los niños no tiene capacidad para hacerlo solo le ayudara un monitor, (ayudándole a sujetarlo, pasándole la mano, etc. )

4. Se les pide que lo pongan delante de ellos y con la ayuda de los adultos se ampliará el círculo para que no choquen unos con otros. El profesor comprueba que la distancia y la posición del aro sea el correcto.

5. A continuación mandamos que vayan metiendo dentro del aro diferentes partes del cuerpo: la cabeza, una pierna, un brazo. El maestro siempre hará una escenificación para que puedan imitarlo.

a. Variante 1: Si el nivel de comprensión lo permite, podemos decirle a algún alumno o alumnos que decidan ellos partes del cuerpo.

b. Variante 2: Con aquellos niños/as que ya conocen las diferentes partes del cuerpo y su vocabulario se intenta trabajar la lateralidad y los conceptos derecha/izquierda (brazo derecho/izquierdo, pierna derecha/izquierda).

c. Variante 2: Si alguno de los niños no tiene capacidad para realizar la tarea según la Variante 1, entonces uno de los tutores procederá a colocarle el aro en la cabeza, la mano, la pierna, etc. complementando la actividad con más tareas de experimentación con sus compañeros y adulto.

6. Si el grupo o la mayor parte del grupo tiene capacidad, el maestro hará una escenificación de cómo se puede hacer girar el aro con la ayuda de las manos y como se puede hacer girar por el suelo y pedirá que intenten imitarlo.

a. Variante 1: Si alguno de los niños no tiene capaci- 
dad para realizar la tarea, un monitor le ayudará a hacerlo girar o le hará girar el aro alrededor de su brazo o de su perna, etc. y después le ayudará a hacerlo girar por el suelo o lo hará girar alredor de él.

7. A continuación dejaremos unos minutos para que experimenten con el aro libremente.

8. Para acabar la sesión pedimos que retornen los aros a su sitio y que formen nuevamente el semicírculo, para poder felicitarlos por lo bien que lo han hecho.

9. Finalmente procedemos con los hábitos de higiene generales establecidos para después de cada clase.

\section{Sesión: 3}

Duración: 50/60 minutos.

Equipo docente: Un maestro y un monitor.

Alumnos: Grupo de 10-12 alumnos.

Espacio: Pista, sala o pabellón.

Material: Tizas, papel de envolver, lápiz.

Organización: Por parejas.

\section{Desarrollo:}

1. Los participante, de pie o sentados, formarán un semicírculo, todos de cara al maestro.

a. Variante: Si alguno de los niños no tiene capacidad para alinearse en el semicírculo, le ayudará un monitor. Si alguno no puede estar de pie se colocará sentado.

2. Explicamos a los alumnos que para esta actividad tienen que formar parejas.

a. Variante 1: Si tienen capacidad les pedimos que escojan compañero.

b. Variante 2: Si no tienen capacidad para agruparse de forma socialmente aceptable, el maestro les manda como tienen que emparejarse.

c. Variante 3: Si algún niño no puede hacer la actividad de forma autónoma, se le asigna un adulto para que lo ayude.

3. Se reparten las parejas por la pista o sala asignándoles un espacio propio.

a. Variante: Si se trabaja en una sala donde no se puede pintar el suelo podemos hacer servir papel grande de envolver, papel de periódico pegado con celo, etc. y utilizar rotuladores, colores, etc. vigilando que no se ensucien la ropa.

4. Se les explica que uno se tiene que tumbar en el suelo y que el otro tiene que dibujar su silueta.

5. El profesor, que ira con un alumno o una pareja de alumnos (a poder ser los alumnos con más dificultades de comprensión) hará la escenificación. Le pedirá a uno de los alumnos que se estire en el suelo o le ayudará a estirarse, estirando brazos y piernas y con una tiza empezará a dibujará su contorno para, si es posible, pedir al compañero que termine de dibujarlo.

6. Pedirá a los niños que vayan a sus sitios e intenten hacer lo mismo. Los adultos irán de grupo en grupo ayudándoles en la tarea.

7. Cuando el primero de ellos acabe, el compañero que está en el suelo intercambiara el rol con el, y tendrá que dibujar su silueta. De esta forma los dos tendrán su silueta dibujada en el suelo.

8. Cuando acaben los adultos ayudarán a los alumnos a repasaran las silueta que no acaben de tener la forma correcta.

9. A continuación reunimos al grupo en el centro del campo vigilando no pisar las siluetas del suelo.

10. Entonces el profesor les explicaque tienen queseguirlo e imitarlo en los desplazamientos que hará por la pista y cuando diga una parte del cuerpo todos tienen que ir a tocar en su silueta la parte indicada. Por ejemplo: La cabeza, el brazo, la pierna, la mano, el pie, etc.

a. Variante: Los adultos vigilaran al grupo ayudándolos a encontrar su silueta o, en caso necesario, llevándolos hasta ella, señalándoles que deben tocar para que ellos lo toquen, ayudándoles a tocarla (visión reducida) o bien tocándola ellos mismos (niños en silla de ruedas).

b. Variante 2: Con aquellos niños/as que ya conocen las diferentes partes del cuerpo y su vocabulario se intenta trabajar la lateralidad y los conceptos derecha/izquierda (brazo derecho/izquierdo, pierna derecha/izquierda).

11. En caso de haber usado papel para dibujar la silueta, para finalizar, tenemos la opción de colgar las siluetas en el aula o de enrollarlas y prepararlas para que se las lleven a casa.

12. Finalmente procedemos con los hábitos de higiene generales establecidos para después de cada clase.

\section{Evaluación}

Evaluación continuada, a partir del diario de aula, empleando como procedimiento la observación directa. Como fase previa se efectúa una evaluación inicial para conocer el alcance de los conocimientos y competencias de los alumnos en relación a los contenidos y objetivos de esta unidad. A continuación, durante las sesiones se irá observando y anotando los aspectos y actitudes destacables de cada alumno, así como las medidas correctivas llevadas a cabo en el proceso de enseñanza y aprendizaje en caso de ser necesarias. Al finalizar de la Unidad Didáctica se valora si se han conseguido los objetivos y si se han adquirido o no los contenidos establecidos. 


\section{Resultados}

En base a los resultados de evaluación puede decirse que existe una relación directa entre la consecución de los objetivos curriculares directamente relacionados con la educación física y el grado de discapacidad.

En general, todos los alumnos responden bien a la metodología de mando directo. Los alumnos con grados más moderados de discapacidad psíquica han sido también capaces de responder bien a metodologías basadas en el descubrimiento guiado, y la experimentación libre, aunque en el segundo caso no siempre se consigue que el alumno centre sus esfuerzos en los objetivos de la actividad.

El trabajo cooperativo ha sido aplicado con resultados variables y muy condicionados por el entorno y el estado emocional de cada uno de ellos. A pesar de ello, en general, se ha conseguido motivar a los alumnos y hacer que participen y disfruten de las actividades lo cual ha generado mas respuestas emocionales positivas que negativas, hecho que ha facilitado la aplicación de estas metodologías y trabajar la cooperación y la socialización.

Durante las actividades se consigue que los alumnos muestren un deseo de llevar a cabo los ejercicios, pero no siempre lo consiguen. La capacidad de aprender es en general limitada, lo cual, excepto en los casos severos de discapacidad, se ve compensada por la capacidad de reconocer y de imitar los gestos y movimientos básicos de las diferentes actividades, lo cual permite trabajar con la mayoría de los alumnos la mayor parte de las actividades motrices planificadas. Solo un número muy reducido de alumnos, con un grado menor de discapacidad, son capaces de aprender y reconocer todas las partes del cuerpo y de llevar a cabo ejercicios de lateralidad y desplazamiento coordinado o de investigar por si solo nuevas interacciones, además de las enseñadas, con los materiales pertenecientes a cada actividad. Ahora bien, en general, las actividades han sido de su agrado, lo cual se ve reflejado en un mayor número de respuestas emocionales positivas a nivel individual y con respecto a sus compañeros y escasos enfrentamientos por la atención del profesor o tutor, por el reparto o tenencia de los materiales de las actividades o por el dominio del espacio. Estos resultados por si solos compensan la escasa respuesta de algunos de los alumnos a las partes más técnicas.

\section{Discusión}

Nuestro objetivo era adaptar una unidad didáctica de Educación Física para un Centro de Educación Especial, ejecutarla y valorar su viabilidad. Llegados a este punto podemos darlo por conseguido.

Las sesiones se han aplicado a tres grupos de niños muy heterogéneos y representativos del centro escolar. La parte más destacable de la experiencia ha sido el haber podido llevarlas a cabo con total normalidad, siguiendo en todo momento la planificación de aula y sin salirnos de los tiempos estipulados para cada actividad, con una duración aproximada de 50 minutos cada una de ellas. Cabe destacar que en todas las sesiones se pudo contar con la ayuda de un maestro de soporte.

El alcance de la consecución de los objetivos didácticos ha dependido del grado de discapacidad de cada alumno, pero en todos los casos se ha observado un progreso más o menos significativo.

Por lo general, todos los alumnos se han ido acostumbrando a las sesiones y a los ejercicios propuestos. Esta aclimatación se puede observar en cómo van aumentando los niveles de motivación y participación por parte de los niños y niñas. Además, entendemos que esta familiarización se produce gracias a que se sigue un tipo de sesión estructurada que repite un patrón fijo (formar semicírculo, decir brevemente que se va a hacer, escenificar, etc.), que ellos ya conocen de unidades didácticas anteriores, que posibilita que los alumnos se centren en la clase, ya que conocen su estructura, y esto les aporta tranquilidad. Hemos observado también como el nivel de participación de la mayoría de los alumnos ha ido aumentando a medida que la Unidad Didáctica ha avanzado. Los alumnos que no consiguen aumentar su participación se caracterizan por presentar un grado y un tipo de discapacidad invalidante, solo participan en ejercicios de mando directo y con un profesor trabajando con ellos individualmente, aunque esto no siempre funciona, ya que hay días que el alumno no se interesa por nada. En estos casos solo es posible valorar el grado de socialización y la reacción positiva a la presencia de sus compañeros y tutores.

En cuanto al desarrollo de habilidades motrices básicas, es una cuestión más individual, unos progresan más rápido que otros y es muy difícil de valorar en una unidad didáctica. En nuestro caso, la valoración se hará a final de curso a partir del diario de aula, donde se anota la evolución de los alumnos. Aun así, en algunos alumnos, la mejora se puede observar día a día y está muy relacionada con la familiarización que se tenga de los contenidos trabajados (si se ha trabajado en cursos anteriores, fuera de la escuela, en anteriores unidades, etc).

En cuanto a objetivos mas específicos como el de explorar y experimentar de forma autónoma diferentes movimientos y posibilidades motrices, con material o sin él, o la adquisición del concepto derecha/izquierda, solo ha podido ser evaluado en aquellos niños que ya tenían un conocimiento previo de las diferentes partes del cuerpo y cierta autonomía personal. El concepto derecha/izquierda solo lo tienen interiorizado dos alumnos entre los tres grupos. Consideramos que es un contenido que se ha de trabajar de manera transversal en todas las asignaturas y añadirlo de manera sutil en próximas unidades didácticas de educación física. Es posible, que aun así, bastantes alumnos nunca lleguen a adquirir este contenido. 
En cuanto a las limitaciones del estudio, encontramos que cada alumno tiene un grado y tipo de discapacidad diferente por lo que su progreso depende de forma directa e éste hecho. Por ello, mientras algunos alumnos van evolucionando a más, otros debido a su discapacidad, se mantendrán en el mismo nivel. Otra de las limitaciones es el bajo número de sesiones por Unidad Didáctica ya que estos niños precisan de más tiempo para cada tarea, pero no es adecuado alargar el tiempo, pues se aburren y se cansan con mucha facilidad. Es importante recalcar que otra limitación durante las clases de Educación Física es la necesidad de contar con más de un docente para poder atender a las necesidades especiales de cada individuo.

En cuanto a la metodología, podemos decir que lo importante es realizar ejercicios sencillos y con objetivos alcanzables donde puedan participar todos los alumnos. Llegados a este punto, el uso de actividades lúdicas variadas, para evitar el aburrimiento y la perdida de atención, con un objetivo constante y repetitivo (conocer e identificar las partes del cuerpo y su vocabulario), junto a un refuerzo positivo constante, conduce poco a poco a la obtención de mejoras. Tal y como se indica en el apartado de resultados, los alumnos responden bien a metodologías de mando directo, pero solo aquellos con grados más moderados de discapacidad psíquica son capaces de responder a metodologías basadas en el descubrimiento guiado, y la experimentación libre. A pesar de ello estas metodologías no han sido descartadas en alumnos con discapacidad psíquica más severa, al haber observado que pueden ser muy prácticas para presentarles futuras actividades que después se trabajarán de forma más dirigida. De esta forma, cuando se plantea la actividad el alumno ya tiene una experiencia previa que hace que le sea más fácil realizar la actividad que se proponga en ese momento.

\section{Conclusiones}

Esta experiencia nos demuestra que si se pueden programar y planear actividades para este tipo de colectivo aunque cada individuo presente unas características diferentes. Para conseguirlo es necesario conocer a los alumnos y sus capacidades, sin olvidar que no todos aprenden de la misma forma, por lo que, para alcanzar una mayor inclusión es necesario conocerlos y saber cuales son los métodos que mejor se adaptan a cada uno de ellos.

\section{Referencias bibliográficas}

1. Alba, J. G., \& Pérez, J. A. P. (2011). Lateralidad en síndrome de Down en edad infantil y adulta. Estudio comparativo. Revista de Psicología y Educación, 1(6), 57-68.

2. Alba, L. A. N., \& Díaz, Á. J. G. (2016). Estilos De Enseñanza Y Tiempo Efectivo De Trabajo En La Categoría Infantil En Creativo Fc. Revista Digital: Actividad Física y Deporte, 1(2).

3. Alonso, M. Á. V. (2003). Análisis de la definición de discapacidad intelectual de la Asociación Americana sobre Retraso Mental de 2002. Siglo cero: Revista Española sobre discapacidad intelectual, 34(205), 5-19.

4. Alós, J. G. (1997). Investigación y prospectiva en educación especial: el diagnóstico. Educar, (21), 85-103.

5. Amate, M. D. M. P., \& Ordás, R. P. (2012). Propuesta de unidad didáctica sobre geocaching: en busca del tesoro escondido. EmásF: revista digital de educación física, (19), 155-172.

6. Ancona, F. E., Heredia, M. C., \& Gómez-Maqueo, E. L. (2016). Psicodiagnóstico clínico del niño (3a edición) México: Editorial El Manual Moderno.

7. Antequera, M., Bachiller, B., Calderón, M. T., Cruz, A., Cruz, P. L., García, F. J., Luna, M., Montero, F., Orellana, F. M. y Ortega, R. (2008). Manual de atención al alumnado con necesidades especificas de apoyo educativo derivadas de la discapacidad intelectual. Sevilla: Junta de Andalucía. Consejería de Educación. Dirección General de Participación e Innovación Educativa. Extraído el 2 de Enero 2016, de http://www.juntadeandalucia.es/educacion/webportal/web/escuelade-familias/neae/necesidades-educativas-especiales/discapacidad-intelectual

8. Arteaga, M., Viciana, V. \& Conde, J. (1997). Desarrollo de la expresividad corporal. Tratamiento globalizador de los contenidos de representación. Barcelona, España: INDE Publicaciones.

9. Barrón, R. G., \& Castilla, I. M. (2016). Psicología clínica infanto-juvenil. Madrid: Pirámide

10. Castañer, M., \& Camerino, O. (1991). La educación física en la enseñanza primaria. Barcelona: INDE Publicaciones.
11. Castro Mangas, A. (2008). Juegos para educación física: desarrollo de destrezas básicas. Madrid: Narcea Ediciones.

12. Fernández, J.J (2011). La actividad físico-deportiva como base para la integración en el área de educación física: autistas. EmasF, Revista Digital de Educación Física. (8), 1-8.

13. García, E., \& Ruiz, F. (2001). Educación Física a través del juego. Primaria. Materiales no convencionales. Propuestas de juegos con globos, cuerdas, papeles, envases, saquitos. Madrid: Gymnos.

14. Gento, S (2011). Tratamiento educativo de la diversidad intelectual. Madrid: Universidad Nacional de Educación a Distancia

15. Iudicibus, L.L (2011). Trastornos generalizados del desarrollo. Alcmeon, Revista Argentina de Clínica Neuropsiquiatría. 17(1), 5-41.

16. Kelly, L. (2006). Adapted Physical Education National Standards. Champaign, IL: Human Kinetics.

17. Le Boulch, J. (1987). La educación psicomotriz en la escuela primaria: la psicokinética en la edad escolar. Barcelona: Paidós

18. Llario, M. D. G., Mengual, V. M., Rodríguez, I. D., García, C. G., \& Palomino, E. R. (2016). Calidad de vida de los discapacitados intelectuales valorada por ellos mismos y los profesionales: variables implicadas. International Journal of Developmental and Educational Psychology. INFAD Revista de Psicología., 1(1), 265-274.

19. Luckasson, R., Borthwick-Duffy, S., Buntinx, W. H., Coulter, D. L., Craig, E. M. P., Reeve, A., Tasse, M. J. (2002). Mental retardation: Definition, classification, and systems of supports. American Association on Mental Retardation. Madrid: Alianza Editorial

20. Márquez, J. M. C., \& Celis, C. C. (2016). Metodología de la actividad física. Sevilla: Wanceulen SL.

21. Novell R, Rueda P, Salvador L, Forgas E. (2003) Salud Mental y alteraciones de la conducta en las personas con discapacidad intelectual. Guía práctica para técnicos y cuidadores. Madrid: Colección FEAPS.

22. Pérez, G. M., \& Martín, J. J. B. (2016). ¿Qué es la innovación docente?: un cambio en las practicas o de pensamiento docente. EmásF: revista digital de educación física, (38), 7-17. 
23. Sanz, D., y Reina, R. (2012). Actividades físicas y deportes adaptados para personas con discapacidad. Barcelona: Paidotribo.

24. Segura, M. J. M. (2011). Formación de maestros, atención educativa a alumnos con plurideficiencia y estimulación sensoriomotriz. Revista electrónica interuniversitaria de formación del profesorado, 14(1), 137-150. 25. Sos, A. A., Clemente, J. A. J., \& González, L. G. (2013). Adaptación del currículum ordinario de Educación Física en Educación Primaria y propuesta metodológica para alumnado escolarizado en centros de Educación Especial. Agora para la educación física y el deporte, 15(3), 228-242.

26. Utley, A. (1994). Enseńanza de la educación física y necesidades educativas especiales. Revista Española de Educación Física y Deportes, 1(4), 24-29. 